\title{
Article
}

\section{Ordinal Time Series Forecasting of the Air Quality Index}

\author{
Cathy W. S. Chen *(1) and L. M. Chiu
}

Department of Statistics, Feng Chia University, Taichung 407, Taiwan; m0703432@mail.fcu.edu.tw

* Correspondence: chenws@mail.fcu.edu.tw

check for

updates

Citation: Chen, C.W.S.; Chiu, L.M. Ordinal Time Series Forecasting of the Air Quality Index. Entropy 2021, 23, 1167. https://doi.org/10.3390/ e23091167

Academic Editor: Reik Donner

Received: 29 June 2021

Accepted: 1 September 2021

Published: 4 September 2021

Publisher's Note: MDPI stays neutral with regard to jurisdictional claims in published maps and institutional affiliations.

Copyright: (c) 2021 by the authors. Licensee MDPI, Basel, Switzerland. This article is an open access article distributed under the terms and conditions of the Creative Commons Attribution (CC BY) license (https:// creativecommons.org/licenses/by/ $4.0 /)$.
Abstract: This research models and forecasts daily AQI (air quality index) levels in 16 cities/counties of Taiwan, examines their AQI level forecast performance via a rolling window approach over a one-year validation period, including multi-level forecast classification, and measures the forecast accuracy rates. We employ statistical modeling and machine learning with three weather covariates of daily accumulated precipitation, temperature, and wind direction and also include seasonal dummy variables. The study utilizes four models to forecast air quality levels: (1) an autoregressive model with exogenous variables and GARCH (generalized autoregressive conditional heteroskedasticity) errors; (2) an autoregressive multinomial logistic regression; (3) multi-class classification by support vector machine (SVM); (4) neural network autoregression with exogenous variable (NNARX). These models relate to lag-1 AQI values and the previous day's weather covariates (precipitation and temperature), while wind direction serves as an hour-lag effect based on the idea of nowcasting. The results demonstrate that autoregressive multinomial logistic regression and the SVM method are the best choices for AQI-level predictions regarding the high average and low variation accuracy rates.

Keywords: ARX-GARCH model; autoregressive logistic regression; artificial neural network; machine learning; support vector machine; multi-class classification; one-step-ahead forecast; training and validation

\section{Introduction}

Monitoring air quality is important for human health and the environment. The well-known air quality index (AQI) presents the status of daily air quality and shows the degree of air pollution and how certain factors affect people's health. This index covers five major air pollutants: (1) ground-level ozone; (2) particle pollution (known as particulate matter, including $P M_{2.5}$ and $P M_{10}$ ); (3) carbon monoxide; (4) sulfur dioxide; (5) nitrogen dioxide. The higher the AQI level is, the greater is the degree of air pollution, and the more serious its impact is on the health of human beings. The U.S. Environmental Protection Agency (EPA) typically employs an AQI value of 100 as the benchmark for air quality to help protect people's health. With reference to U.S. standards, Taiwan's AQI includes an 8-h moving average of ozone, an hourly average of ozone, a 12-h weighted average of $P M_{2.5}$, a 12-h weighted average of $P M_{10}$, an 8-h average of carbon monoxide, hourly average of sulfur dioxide, and hourly average of nitrogen dioxide. With a total of seven indicators, the most serious index value then denotes the value of AQI. The U.S. EPA classifies AQI values into six levels, and each level corresponds to different health problems; see Table 1.

Predicting AQI classes plays an important role in issuing health alerts when AQI levels exceed the specified levels. This study thus aims to build a forecasting system based on the level of AQI rather than AQI values. Categorical time series can be either nominal or ordinal. The time series of AQI levels is ordinal in the sense that the class increases as the AQI interval value increases. Some studies in the literature focus on forecasting AQI values or individual pollutant concentrations based on neural network, mode decomposition, and ARIMA (autoregressive integrated moving average) models; e.g., [1-5]. Alternatively, Liu et al. [6] propose a zero-one-inflated bounded Poisson model for air quality level data, while Kim [7] employs a generalized linear model for the ozone level. Our study 
mainly predicts multi-level AQI classifications, which help forecast qualitative AQI at time $t$ based on information up to $t-1$. With respect to the prediction, we should not utilize current information, especially as AQI includes the pollutants $\mathrm{SO}_{2}, \mathrm{NO}_{2}, \mathrm{O}_{3}, \mathrm{CO}$, $P M_{10}$, and $P M_{2.5}$. We instead consider three weather covariates and seasonal variables that could influence the change in AQI, including daily accumulated precipitation (PRE), daily average temperature (TEM), and daily wind direction (WD). Henry et al. [8] use nonparametric regression to determine the relationship between the concentrations of air pollutants and WD. We utilize an hour-lag effect of WD, which is widely used by meteorologists for nowcasting - that is, information of the covariate WD is available up to time $(t+m-1)$, where $m$ is the hour difference between AQI and WD.

Table 1. AQI's six levels based on the U.S. EPA, where each level corresponds to different health problems.

\begin{tabular}{lll}
\hline Value & Color & AQI Levels of Health Concern \\
\hline $0-50$ & Green & Good \\
$51-100$ & Yellow & Satisfactory \\
$101-150$ & Orange & Moderately \\
$151-200$ & Red & Poor \\
$201-300$ & Purple & Very poor \\
$301-500$ & Maroon & Hazardous \\
\hline
\end{tabular}

As AQI relates to its own historical information [9], we utilize the following statistical modeling and machine learning approaches with the three weather covariates and seasonal dummy variables for the predictions. First, we employ an autoregressive model with the above-mentioned weather covariates (commonly called exogenous variables) and GARCH (generalized autoregressive conditional heteroskedasticity) errors if heteroscedasticity exists in the time-series data; see So et al. [10] and Chen et al. [11]. The ARCH and GARCH models originally appear in Engle [12] and Bollerslev [13] and have been widely used in modeling the conditional second-moment properties of time-series data. We observe that the time series of AQI values exhibit conditional heteroskedasticity and autocorrelation in the squared series, which are said to have ARCH effects. We then predict one-stepahead AQI values and convert them into the AQI level based on this proposed model, which we name the ARX-GARCH model. Here, " $X$ " stands for those covariates and seasonal variables.

Second, we employ an autoregressive multinomial logistic regression for the AQI ordinal response variable, for which this model includes the lag-1 AQI value, the weather covariables, and seasonal variables. This model is an excellent tool for analyzing binary and ordinal data, allowing us to estimate effects and to make predictions [14]. We call this model the AR logistic regression for short hereafter. Some popular classifiers to forecast pollution are neural networks [15] and support vector machines (SVM) [16,17]. SVM, a type of machine learning technique, is a supervised learning model with related learning algorithms that analyze data used for binary and multi-level classifications.

Third, we use the SVM method with the same covariates as in the AR logistic regression for classification and prediction. Liu et al. [17] utilize the current effects of $P M_{2.5}$, $\mathrm{PM}_{10}, \mathrm{SO}_{2}, \mathrm{CO}, \mathrm{NO}_{2}$, and $\mathrm{O}_{3}$ as input variables. On the contrary, our study excludes these current effects for the forecast purpose. We instead incorporate the lag-1 AQI value in the forecasting models since AQI covers the five major air pollutants, which simultaneously include many lags of the major pollution concentrations.

Fourth, we employ the neural network autoregression with exogenous variable (NNARX) model, which is capable of recognizing, classifying, or predicting datasets. Applications of autoregressive neural network modeling have been successfully predicting time-series datasets; e.g., Hertz et al. [18] and Hyndman and Athanasopoulos [19]. 
This study encounters several missing values in AQI, such as accumulated precipitation, daily average temperature, and wind direction. In order to preserve the characteristics of the time series, we do not remove any missing values directly for information loss and instead adopt $k$-nearest neighbors as proposed by Cover and Hart [20] to fill in the missing values; see Torgo [21] for the $k$-Nearest Neighbors (knn) algorithm. Hence, we implement the $\mathrm{knn}$ imputation to fill in the missing value under different $k$ values with different variables to make the mean absolute error (MAE) and root mean squared error (RMSE) as small as possible.

The rest of this study runs as follows. Section 2 introduces the models for forecasting ordinal time series-the AQI levels. Section 3 shows the data description and provides information about training/validation of the methodology in order to assess the forecast performance. Section 4 provides the AQI level forecasts and evaluates forecast accuracy. Finally, Section 5 offers concluding remarks.

\section{Models and Methods}

To predict the one-step-ahead AQI level, we describe the four proposed models for the daily prediction as follows. Let $Y_{t}$ be the daily AQI value, $Y_{t-1}$ is the previous day's AQI value, and $\left(X_{1, t}, X_{2, t}, X_{3, t}\right)$ are respectively PRE, TEM, and WD. Let $Y_{t}^{*}, t=1, \ldots, n$ be the observed AQI level at time $t$, with the following $r$ possible outcomes:

$$
Y_{t}^{*}= \begin{cases}j & \text { if the } j t h \text { level is observed at time } t, \\ 0 & \text { otherwise, }\end{cases}
$$

for $j=1, \ldots, r$. The proposed methods consider a seasonal or periodic pattern to improve the prediction via seasonal dummy variables or a monthly indicator.

Model 1: The ARX model with GARCH errors

$$
\begin{aligned}
Y_{t} & =\phi_{0}+\phi_{1} Y_{t-1}+f\left(X_{1, t-1}, X_{2, t-1}, X_{3, t+m-1}\right)+S N_{t}+a_{t}, \\
a_{t} & =\sigma_{t} \varepsilon_{t}, \varepsilon_{t} \stackrel{i i d}{\sim} S t(v, r) \\
\sigma_{t}^{2} & =\alpha_{0}+\alpha_{1} a_{t-1}^{2}+\beta_{1} \sigma_{t-1}^{2}, \\
f(.) & =\gamma_{1} X_{1, t-1}+\gamma_{2} X_{2, t-1}+\gamma_{3} X_{3, t+m-1}, \\
S N_{t} & =\beta_{s 1} D_{s 1, t}+\beta_{s 2} D_{s 2, t}+\ldots+\beta_{s 11} D_{s 11, t}, \\
D_{s i, t} & = \begin{cases}1 & \text { if } t \text { is in month } i \\
0 & \text { otherwise, }\end{cases}
\end{aligned}
$$

where $a_{t}$ is the error term; $\varepsilon_{t}$ follows the skewed Student-t distribution by Hansen [22] since the data are non-normal; and St $(v, r)$ is a skewed Student-t distribution with shape parameter $v$ and skewness parameter $r$. Here, (1) and (2) are respectively the mean equation and the volatility equation. The lag $m$ of $X_{3, t+m-1}$ denotes the time difference in hours between AQI and WD. It is based on the idea of nowcasting. In order to guarantee stationarity and positiveness in volatility, we restrict the parameters as:

$$
\begin{aligned}
& S 1:\left|\phi_{1}\right|<1 \\
& \text { S2: } \alpha_{0}>0, \alpha_{1}, \beta_{1} \geq 0, \alpha_{1}+\beta_{1}<1 \text {. }
\end{aligned}
$$

As the ARX-GARCH models in (1) are designed for continuous dependent variables, we obtain the one-day-ahead forecast based on the ARX-GARCH model and then convert the forecasts into the AQI level as in Table 1. 
Latent variable models with observed ordinal variables are particularly useful for analyzing the AQI levels. Next, we consider an AR logistic regression for $Y_{t}^{*}$.

Model 2: The AR logistic regression

The conditional probability for the AQI level at day $t$ is:

$$
\begin{aligned}
& \operatorname{Pr}\left(Y_{t}^{*}=i \mid Y_{t-1}, X_{1, t-1}, X_{2, t-1}, X_{3, t+m-1}\right)= \\
& \frac{\exp \left(g_{i}\left(Y_{t-1}, X_{1, t-1}, X_{2, t-1}, X_{3, t+m-1}, S N_{t}\right)\right)}{1+\sum_{j=1}^{r-1} \exp \left(g_{j}\left(Y_{t-1}, X_{1, t-1}, X_{2, t-1}, X_{3, t+m-1}, S N_{t}\right)\right)},
\end{aligned}
$$

where $g_{i}($.$) is a linear function with i=1, \ldots, r-1$. Apart from this model, Weiß [23] and subsequently Liu et al. [6] propose two new methods for dealing with ordinal time series; e.g., the rank-count approach for time series modeling.

Model 3: The SVM classification method

SVM classification is an optimization problem, which is a machine learning algorithm for the dependent variable, isolating the group to which it should belong. If the subset trained in two dimensions is a linearly separable set, then it means that the whole set itself is linearly separable. We also can use SVM for classifying a non-linear dataset and conduct the classification by projecting the dataset into a higher dimension in which it is linearly separable. We consider multi-dimensions, where the dividing line becomes a separating hyperplane and separates into more than two different levels. The hard-margin must be met so that the distance between the separating hyperplane and each group is equal; in other words, the margins are maximized. We use the SVM method, and the hyperplane $h(\boldsymbol{X})$ is:

$$
\begin{aligned}
h(X) & =\alpha+\phi_{1} Y_{t-1}+f\left(X_{1, t-1}, X_{2, t-1}, X_{3, t+m-1}\right)+\beta_{4} M_{t}, \\
M_{t} & = \begin{cases}i & \text { if } t \text { is in month } i \\
0 & \text { otherwise, }\end{cases}
\end{aligned}
$$

where $f($.$) is the same as in Equation (3). The algorithm creates a line or a hyperplane$ that separates the data in the training (or learning) period into classes. Each hyperplane $h(\boldsymbol{X})$ that is trained by the learning period divides the validation period into specific levels and gives a score based on their proximity to the hyperplane. We execute the final step of classifications by assigning the validation period to the level from which they obtain the highest score.

Model 4: We consider the NNARX model, feed-forward networks, and two hidden layers and use the notation $\operatorname{NNARX}(1,2)$. This model includes the lag- $1 \mathrm{AQI}$ value and $\left\{X_{1, t-1}, X_{2, t-1}, X_{3, t+m-1}\right\}$ as exogenous variables. The NNARX model with hidden layers achieves greater forecasting accuracy, but the cost is a large computation time as the number of hidden layers increases.

\section{Data Collection}

Particulate pollutants originating from the Asian continent, in particular eastern China, are often carried to Taiwan along with the monsoons; see Hsu et al. [24]. This means the WD covariate plays a significant role in the prediction of the AQI level. The other pollutants in Taiwan mainly come from domestic combustion such as the burning of fossil fuels (industrial emissions). The remainder comes from traffic emissions and other air pollution sources. The industrial centers and business parks along the northern and western coasts of Taiwan are surrounded by high mountains, which lead to poor avenues of dispersement and can trap pollutants [24].

Chen et al. [25] support the causal relationship between weekly influenza cases and weekly adjusted accumulative $P M_{2.5}$ in the central and southern regions of Taiwan for various age groups. Traffic-related emissions and coal combustion are the sources of toxic metals in $P M_{10}$ and $P M_{2.5}$, respectively, leading to high risks of cancer [24]. Tseng et al. [26] 
provide epidemiological evidence that more than $50 \%$ of all patients with lung cancer had never smoked and present a positive association between lung cancer and $P M_{2.5}$ exposure in Taiwan, while Tang et al. [27] demonstrate that air pollution in Taiwan, represented by $P M_{2.5}$ or PSI (Pollutant Standards Index), moderately correlates with the development of atopic dermatitis in adults.

Wide differences in air pollution trends have existed between northern and southern Taiwan for decades [26]. We thus investigate daily AQI from the monitoring stations of 16 cities/counties in Taiwan from north to south on the country's west coast, whose names are listed in Table 2 and their geographical locations appear in Figure 1. Our dataset covers a total of 1227 observations from 30 November 2016 to 9 April 2020 at each monitoring station and includes daily data for AQI, $P M_{2.5}$, PRE, TEM, and WD for the 16 cities/counties from EPA, Executive Yuan, Taiwan. The training period includes 852 observations (from 30 November 2016 to 31 March 2019), and we use the last 375 days (from 1 April 2019 to 9 April 2020) as the validation period via a rolling window approach. In order words, we choose a rolling sample size of 852 for parameter estimation (or classification) in the training period and forecast the one-day-ahead AQI level for the validation period. The mechanism of the rolling window approach appears in Figure 2. We measure WD in units from $0^{\circ}$ to $360^{\circ}$. When the wind speed is $\leq 0.2 \mathrm{~m} / \mathrm{s}$, we record WD as $0^{\circ}$ and the north wind as $360^{\circ}$. As the variable WD (in degrees) itself does not have any physical meaning, we classify wind directions in sixteen sectors in Table 3, making it a quantitative variable.

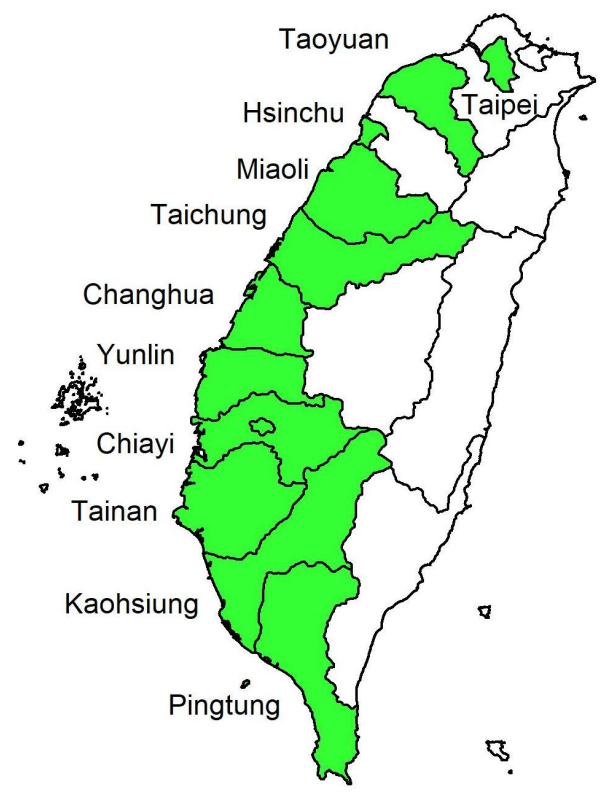

Figure 1. Geographical locations of the 16 sites in Taiwan. Taipei: site 1; Taoyuan: site 2; Hsinchu: site 3; Miaoli: site 4; Taichung: sites 5 and 6; Changhua: site 7; Yunlin: site 8; Chiayi: sites 9 and 10; Tainan: sites 11 and 12; Kaohsiung: sites 13 and 14; Pingtung: sites 15 and 16.

Some missing values are present in the dataset that are often encountered in practice. The percentages of missing values for AQI are between $0.97 \%$ and $2.09 \%$. A small portion of missing values also occurs in the weather covariates. When we deal with a tiny proportion of missing values, we still need to tackle this problem in time series data. We employ the book-associated R package called "DMwR" in Torgo [21] to impute the missing values. Due to limited space, we summarize how we handle missing value imputation below.

(1) PRE or TEM with missing values: If PRE (TEM) is missing at day $t$, then we select the valid data of the nearest station to impute the missing value. 
(2) AQI with missing values: We use $k=4$ in the knn algorithm in terms of the smallest MAE and RMSE to impute the missing AQI value. We first identify the four days with PRE, TEM, WD, $P M_{2.5}$, and seasonal dummy values closest to the day with missing AQI, and the missing AQI is then substituted by the weighted average of the AQI values of these four days. The weights decrease as the distance of the case to its neighbor lengthens. We use a Gaussian kernel function to obtain the weights from the distances and refer for a detailed description of the distance to Torgo [21] on pages 61-62.

(3) WD with missing values: We again use $k=4$ in the knn algorithm to impute the missing WD value. We first identify the four days with PRE, TEM, AQI, $P M_{2.5}$, and seasonal dummy values closest to the day with missing WD, and the missing WD is then substituted by the weighted average of the WD values of these four days.

In order to better understand the characteristics of our datasets, Table 2 displays descriptive statistics of AQI values for each site, which include mean, standard deviation (std), minimum, maximum, and $p$-values of the ARCH test. All averages of AQI values are above 50 except for Hengchun (44.8 at site 16), as Hengchun Peninsula in Pingtung County is considered to have clean air. Kaohsiung and Pingtung (sites 13, 14, and 15) have the three worst AQI values among the observed sites in this study. The $p$-values of the Kolmogorov-Smirnov test $\left(H_{0}\right.$ : The series is from a normal distribution) are all less than 0.0001 , indicating that the AQI data do not follow a normal distribution.

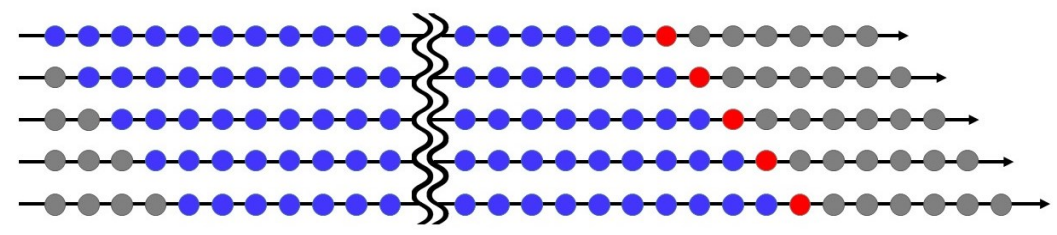

Figure 2. The rolling window approach

Table 2. Descriptive statistics of daily AQI values.

\begin{tabular}{lllllll}
\hline Site & City/County & Mean & Std & Min & Max & $\begin{array}{r}\text { ARCH Test } \\
(\boldsymbol{p} \text {-Value })\end{array}$ \\
\hline 1 & Shilin, Taipei & 54.888 & 23.799 & 13 & 185 & $<0.0001$ \\
2 & Taoyuan, Taoyuan & 54.925 & 24.443 & 15 & 197 & $<0.0001$ \\
3 & Dongqu, Hsinchu & 57.873 & 27.444 & 12 & 203 & $<0.0001$ \\
4 & Miaoli, Miaoli & 61.197 & 25.893 & 13 & 172 & $<0.0001$ \\
5 & Xitun, Taichung & 67.271 & 32.275 & 19 & 190 & $<0.0001$ \\
6 & Fengyuan, Taichung & 62.861 & 29.702 & 15 & 207 & $<0.0001$ \\
7 & Changhua, Changhua & 65.298 & 31.092 & 15 & 190 & $<0.0001$ \\
8 & Douliu, Yunlin & 80.409 & 38.347 & 1 & 195 & $<0.0001$ \\
9 & Xingang, Chiayi & 74.014 & 34.615 & 6 & 179 & $<0.0001$ \\
10 & West, Chiayi & 75.400 & 36.941 & 16 & 179 & $<0.0001$ \\
11 & West Central, Tainan & 74.512 & 37.539 & 17 & 195 & $<0.0001$ \\
12 & Xinying, Tainan & 74.445 & 34.831 & 15 & 179 & $<0.0001$ \\
13 & Zuoying, Kaohsiung & 81.031 & 42.767 & 17 & 210 & $<0.0001$ \\
14 & Quian, Kaohsiung & 79.332 & 41.196 & 17 & 197 & $<0.0001$ \\
15 & Pingtung, Pingtung & 81.964 & 39.995 & 15 & 206 & $<0.0001$ \\
16 & Hengchun, Pingtung & 43.935 & 21.498 & 3 & 182 & $<0.0001$ \\
\hline & & & & & &
\end{tabular}


Table 3. Wind direction and degree.

\begin{tabular}{crr}
\hline Category & Degree & Direction \\
\hline 1 & $348.75-11.25$ & $\mathrm{~N}$ \\
2 & $11.25-33.75$ & $\mathrm{NNE}$ \\
3 & $33.75-56.25$ & $\mathrm{NE}$ \\
4 & $56.25-78.75$ & $\mathrm{ENE}$ \\
5 & $78.75-101.25$ & $\mathrm{E}$ \\
6 & $101.25-123.75$ & $\mathrm{ESE}$ \\
7 & $123.75-146.25$ & $\mathrm{SE}$ \\
8 & $146.25-168.75$ & $\mathrm{SSE}$ \\
9 & $168.75-191.25$ & $\mathrm{~S}$ \\
10 & $191.25-213.75$ & $\mathrm{SSW}$ \\
11 & $213.75-236.25$ & $\mathrm{SW}$ \\
12 & $236.25-258.75$ & $\mathrm{WSW}$ \\
13 & $258.75-281.25$ & $\mathrm{~W}$ \\
14 & $281.25-303.75$ & $\mathrm{WNW}$ \\
15 & $303.75-326.25$ & $\mathrm{NW}$ \\
\hline
\end{tabular}

The ARCH test is a Lagrange multiplier test to assess the significance of ARCH effects. A small $p$-value indicates rejection of the null hypothesis $\left(H_{0}\right.$ : There is no ARCH effect) in favor of the alternative. All $p$-values of the ARCH test are less than 0.0001, which supports the existence of ARCH effects. Heteroscedasticity refers to residuals for an AR model that do not have a constant variance. Therefore, we need to specify the GARCH effect and non-normal errors instead of regular AR models. Table 4 lists descriptive statistics for two weather covariates: PRE and TEM. The standard deviation of "PRE" increases toward the south in general.

Table 4. Descriptive statistics of two daily weather covariates.

\begin{tabular}{llrrrrrrr}
\hline \multirow{2}{*}{ Site } & \multirow{2}{*}{ City/County } & \multicolumn{3}{c}{ PRE } & \multicolumn{4}{c}{ TEM } \\
& & Mean & Std & Max & Mean & Std & Min & Max \\
\hline 1 & Shilin, Taipei & 4.70 & 14.40 & 202.0 & 23.94 & 5.32 & 8.10 & 33.10 \\
2 & Taoyuan, Taoyuan & 5.02 & 15.28 & 175.0 & 22.25 & 5.56 & 6.90 & 32.40 \\
3 & Dongqu, Hsinchu & 4.03 & 14.64 & 260.0 & 23.41 & 5.35 & 7.60 & 31.80 \\
4 & Miaoli, Miaoli & 3.73 & 12.53 & 151.0 & 23.32 & 5.35 & 7.50 & 31.60 \\
5 & Xitun, Taichung & 4.22 & 15.23 & 179.0 & 23.80 & 5.03 & 7.90 & 31.60 \\
6 & Fengyuan, Taichung & 5.17 & 19.28 & 279.0 & 23.20 & 4.84 & 6.40 & 30.80 \\
7 & Changhua, Changhua & 4.54 & 15.97 & 164.5 & 23.38 & 4.63 & 7.80 & 31.20 \\
8 & Douliu, Yunlin & 4.67 & 19.41 & 415.0 & 23.70 & 4.64 & 8.20 & 31.30 \\
9 & Xingang, Chiayi & 3.87 & 16.43 & 332.5 & 23.93 & 4.71 & 9.00 & 31.20 \\
10 & West, Chiayi & 4.85 & 19.32 & 417.0 & 24.35 & 4.59 & 9.20 & 31.70 \\
11 & West Central, Tainan & 5.00 & 21.71 & 373.0 & 24.97 & 4.44 & 9.70 & 31.60 \\
12 & Xinying, Tainan & 4.62 & 20.50 & 451.5 & 24.33 & 4.57 & 9.00 & 31.50 \\
13 & Zuoying, Kaohsiung & 4.62 & 19.24 & 283.5 & 24.97 & 4.03 & 10.20 & 30.90 \\
14 & Qiangin, Kaohsiung & 5.06 & 21.02 & 291.5 & 25.32 & 3.85 & 10.90 & 30.30 \\
15 & Pingtung, Pingtung & 6.44 & 24.92 & 357.0 & 25.14 & 3.57 & 11.00 & 31.20 \\
16 & Hengchun, Pingtung & 5.42 & 20.18 & 326.0 & 25.91 & 3.12 & 15.50 & 31.00 \\
\hline
\end{tabular}

Figure 3 shows the time series plots of daily AQI values (imputation for missing datapoints) for Shilin, Fengyuan, Zuoying, and Pingtung (sites 1, 6, 13, and 15 given in Table 2). These four monitoring stations represent the north, center, and south of Taiwan. Figures 4 and 5 illustrate their related daily PRE and TEM in the same four sites. The time series plots of AQI present obvious seasonal patterns with a gradual decrease in 
May each year. AQIs are relatively low in June and July and gradually increase again until October. The pattern denotes that AQI is lower in summer than in other seasons, and the worst season is winter. We clearly notice that the rainy season is from May to September, and the highest amount of daily accumulated precipitation appears in July and August. Other cities/counties also present similar seasonal patterns. We observe that heavy rain is more likely to occur in the central and southern regions. In addition, the average temperature also shows a seasonal pattern. There are no sixth-level AQI values (301-500) in this study. We present the relative frequencies of AQI levels for each month based on the four monitoring stations noted above in Figure 6. We discover that a larger proportion of the first level (good) appears during summer. "Moderate" and "poor" air quality occurs in summer in a small proportion and then at a higher proportion in spring and winter. We scrutinize larger proportions in levels 2 and 3 (AQI > 100) in Zuoying and Pingtung, which represent south Taiwan.
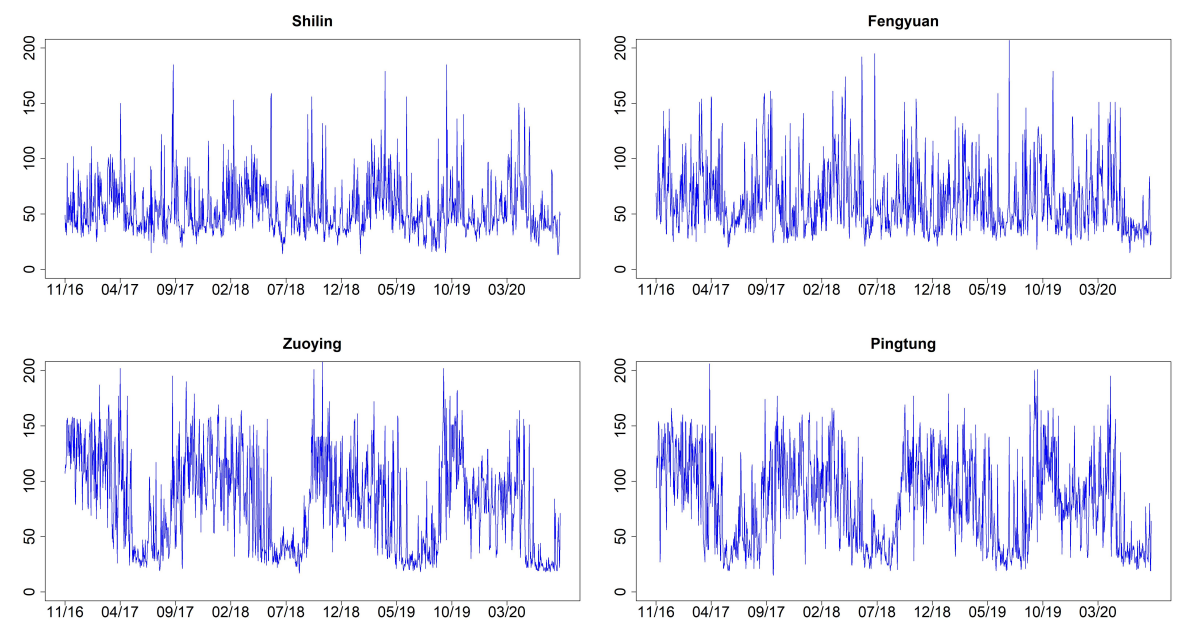

Figure 3. Time series plots of daily AQI values for Shilin, Fengyuan, Zuoying, and Pingtung (sites 1, 6, 13, and 15) from 30 November 2016 to 9 April 2020.
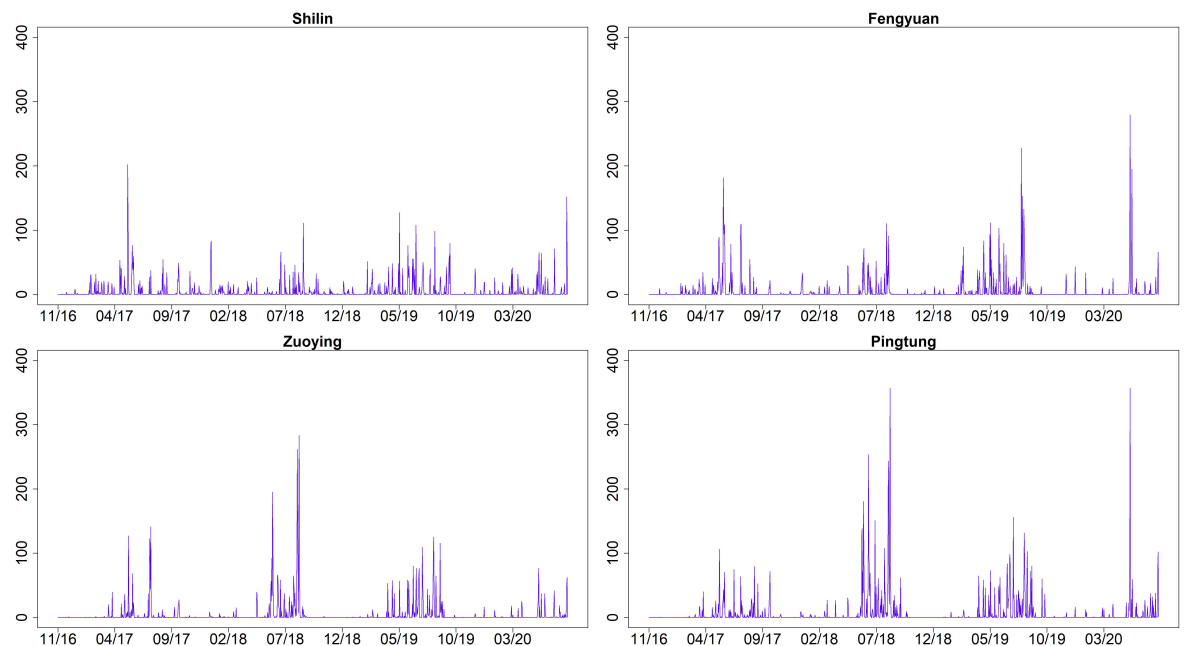

Figure 4. Time series plots of daily PRE for Shilin, Fengyuan, Zuoying, and Pingtung (sites 1, 6, 13, and 15) from 30 November 2016 to 9 April 2020. 


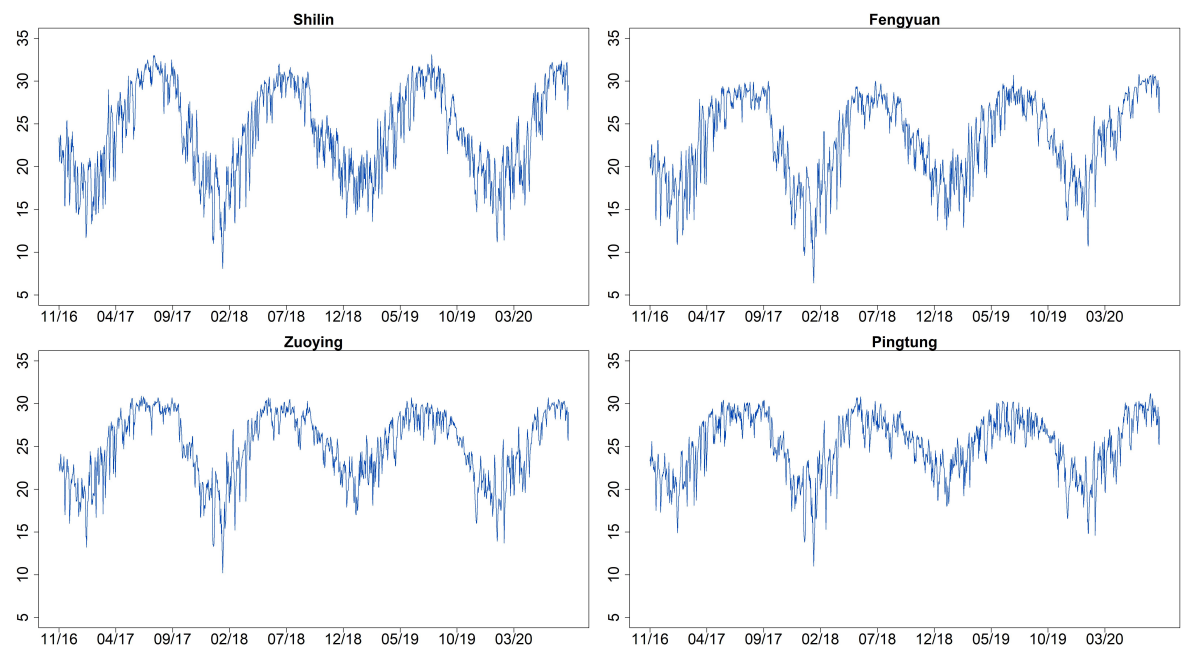

Figure 5. Time series plots of daily TEM for Shilin, Fengyuan, Zuoying, and Pingtung (sites 1, 6, 13, and 15) from 30 November 2016 to 9 April 2020.

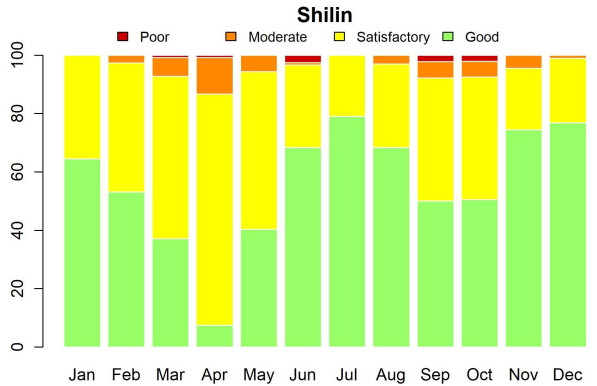

Zuoying

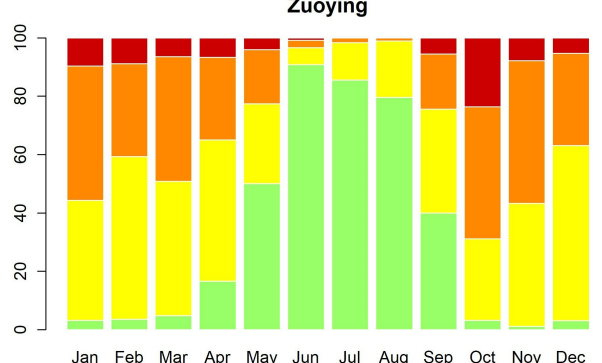

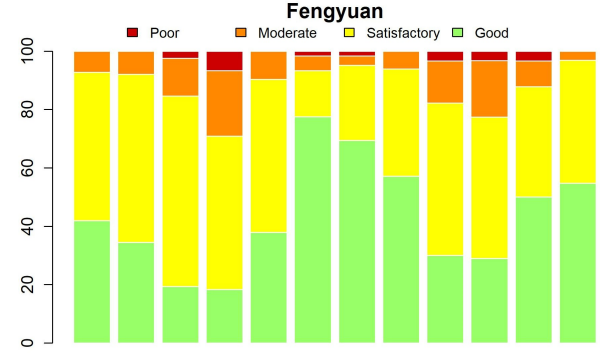

Jan Feb Mar Apr May Jun Jul Aug Sep Oct Nov Dec Pingtung

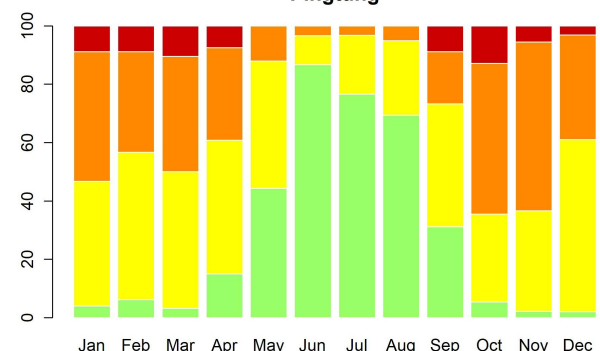

Figure 6. Monthly AQI levels for Shilin, Fengyuan, Zuoying, and Pingtung (sites 1, 6, 13, and 15) from 30 November 2016 to 9 April 2020.

\section{Results}

We predict the one-day-ahead AQI level by using a rolling window approach with a hold-out set: 375 days, which cover just a bit more than one year. We evaluate forecast performance for air quality levels, which include two levels and four levels based on four models: (1) the ARX-GARCH model; (2) the AR logistic regression; (3) the SVM method; (4) the NNARX model.

We add 11 monthly dummy variables as covariates in the ARX-GARCH model and AR logistic regression since the AQI time series presents a seasonal pattern. We use 1 to 12 values of the month indicator variable in the SVM method and NNARX model, because this setup improves the accuracy rate in the AQI-level prediction. We estimate the model parameters and the one-day-ahead forecasts by using the R package "rugarch" [28] for Model 1 (ARX-GARCH model). For the AR logistic regression and SVM method, we use the R package "MASS" [29] and the "e1071" package [30], respectively. For the NNARX model, we employ the R package "neuralnet".

The categorization used for the two-level forecast is based on the threshold 100 (1st level: AQI $\leq 100$; 2nd level: AQI $>100$ ). Table 5 presents the correct classification rates 
for two-level predictions. The accuracy rates of all sites are greater than $81 \%$ for the SVM method. Most accuracy rates by the AR logistic and NNARX models are over $80 \%$, except for site 15 at $78.13 \%$, while the NNARX model has accuracy rates of $79.73 \%$ and $78.40 \%$ for sites 13 and 15, respectively. The ARX-GARCH model does not predict sites 8 , 13,14 , and 15 very well.

We refine the second category into three levels: (i) 101-150 slightly polluted; (ii) 151200 moderately polluted; (iii) 201-300 heavily polluted. When we turn to multi-level forecasting, Table 6 illustrates the percentage accuracy, average, standard deviation (std), and coefficient of variation (CV) of the four models. We show boxplots (a five-number summary with minimum, 1st quantile, median, 3rd quantile, and maximum) for the twolevel and four-level predictions in Figures 7 and 8 . The notation $\triangle$ stands for the average of accuracy rates from all 16 sites for each model. We summarize the results as follows.

1. The overall performances of the AR logistic regression, SVM, and NNARX are similar. Most accuracy rates by the three models are over $80 \%$, except for sites 13 and 15 . The SVM classification method has the highest average accuracy rate $(88.53 \%)$ and the lowest standard deviation (7.00\%) in the four-level predictions. The SVM method and $A R$ logistic regression provide the lowest $C V$ values among the four forecasting techniques. A lower $\mathrm{CV}$ is favored because it provides the most optimal forecasting performance with low variability, but a high average accuracy rate.

2. The prediction performance is relatively low at sites 13 and 15 , and so we need to scrutinize the time series data carefully. We perceive that the major misspecified levels occur in October. The three classification models (AR logistic regression, SVM, and NNARX) tend to misspecify the rare level.

3. The lag-1 weather covariates of PRE and TEM with the hour-lag effect of WD are able to generate a reliable prediction level. We also consider wind speed and weekly/monthly weighted moving averages of AQI as extra covariates. However, these covariates do not improve forecast accuracy. Therefore, we do not include their results in the paper.

4. The ARX-GARCH model is for quantitative forecasting purposes. The classification of wind directions in the sixteen sectors may not be suitable as an explanatory variable for this model.

Table 5. Accuracy based on two forecast levels.

\begin{tabular}{llrrrr}
\hline Site & City/County & ARX-GARCH & $\begin{array}{c}\text { Accuracy (\%) } \\
\text { AR Logistic }\end{array}$ & SVM & NNARX \\
\hline 1 & Shilin, Taipei & 95.20 & 95.20 & 95.47 & 95.47 \\
2 & Taoyuan, Taoyuan & 95.20 & 95.44 & 96.53 & 96.53 \\
3 & Dongqu, Hsinchu & 93.60 & 93.16 & 97.07 & 93.07 \\
4 & Miaoli, Miaoli & 96.00 & 96.71 & 96.80 & 97.33 \\
5 & Xitun, Taichung & 86.13 & 87.85 & 90.93 & 89.07 \\
6 & Fengyuan, Taichung & 88.53 & 89.62 & 88.27 & 89.07 \\
7 & Changhua, Changhua & 91.73 & 91.20 & 94.67 & 94.13 \\
8 & Douliu, Yunlin & 78.13 & 81.52 & 84.27 & 82.93 \\
9 & Xingang, Chiayi & 83.47 & 86.58 & 85.60 & 84.27 \\
10 & West, Chiayi & 81.87 & 85.32 & 85.60 & 88.27 \\
11 & West Central, Tainan & 80.53 & 83.04 & 83.20 & 82.93 \\
12 & Xinying, Tainan & 84.00 & 87.85 & 87.20 & 86.13 \\
13 & Zuoying, Kaohsiung & 78.93 & 82.67 & 81.60 & 79.73 \\
14 & Qianjin, Kaohsiung & 76.80 & 82.40 & 83.47 & 82.13 \\
15 & Pingtung, Pingtung & 73.07 & 78.13 & 82.93 & 78.40 \\
16 & Hengchun, Pingtung & 97.60 & 98.23 & 97.07 & 97.07 \\
\hline
\end{tabular}


Table 6. Accuracy based on four forecast levels.

\begin{tabular}{|c|c|c|c|c|c|}
\hline \multirow{2}{*}{ Site } & \multirow{2}{*}{ City/County } & \multicolumn{4}{|c|}{ Accuracy (\%) } \\
\hline & & ARX-GARCH & AR Logistic & SVM & NNARX \\
\hline 1 & Shilin, Taipei & 95.47 & 95.20 & 95.47 & 95.47 \\
\hline 2 & Taoyuan, Taoyuan & 96.80 & 96.80 & 96.53 & 93.03 \\
\hline 3 & Dongqu, Hsinchu & 93.33 & 93.60 & 97.07 & 93.07 \\
\hline 4 & Miaoli, Miaoli & 97.07 & 97.07 & 96.80 & 97.33 \\
\hline 5 & Xitun, Taichung & 86.67 & 89.07 & 90.40 & 88.80 \\
\hline 6 & Fengyuan, Taichung & 88.27 & 88.80 & 88.27 & 88.80 \\
\hline 7 & Changhua, Changhua & 93.87 & 94.67 & 94.67 & 94.13 \\
\hline 8 & Douliu, Yunlin & 76.27 & 82.13 & 82.13 & 81.07 \\
\hline 9 & Xingang, Chiayi & 84.00 & 85.07 & 85.60 & 84.27 \\
\hline 10 & West, Chiayi & 82.93 & 85.33 & 85.07 & 88.00 \\
\hline 11 & West Central, Tainan & 82.13 & 83.73 & 82.67 & 82.67 \\
\hline 12 & Xinying, Tainan & 86.13 & 87.20 & 86.67 & 85.87 \\
\hline 13 & Zuoying, Kaohsiung & 75.73 & 78.13 & 76.80 & 75.47 \\
\hline 14 & Qianjin, Kaohsiung & 75.20 & 80.53 & 82.40 & 80.53 \\
\hline 15 & Pingtung, Pingtung & 70.13 & 74.40 & 78.93 & 74.93 \\
\hline \multirow[t]{4}{*}{16} & Hengchun, Pingtung & 97.07 & 96.80 & 97.07 & 96.80 \\
\hline & Average (\%) & 86.32 & 88.03 & 88.53 & 87.52 \\
\hline & Std $(\%)$ & 8.825 & 7.182 & 7.000 & 7.234 \\
\hline & $\mathrm{CV}$ & 0.1022 & 0.0816 & 0.0790 & 0.0826 \\
\hline
\end{tabular}

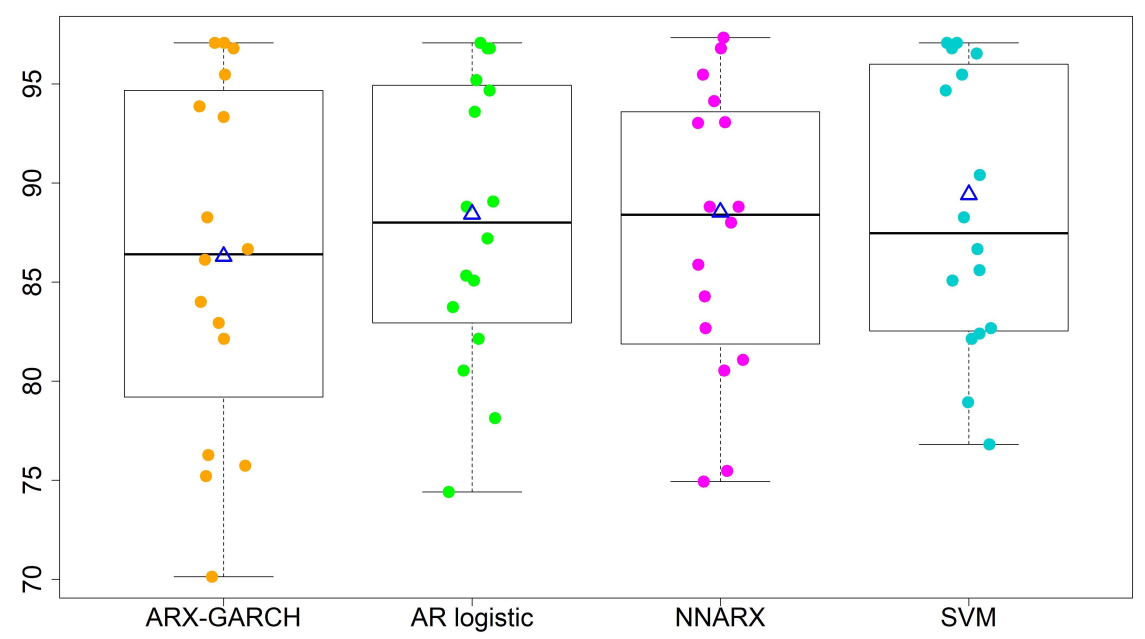

Figure 7. Forecasting performance for two-level classifications. " $\triangle$ "denotes the average of accuracy rates from all 16 sites.

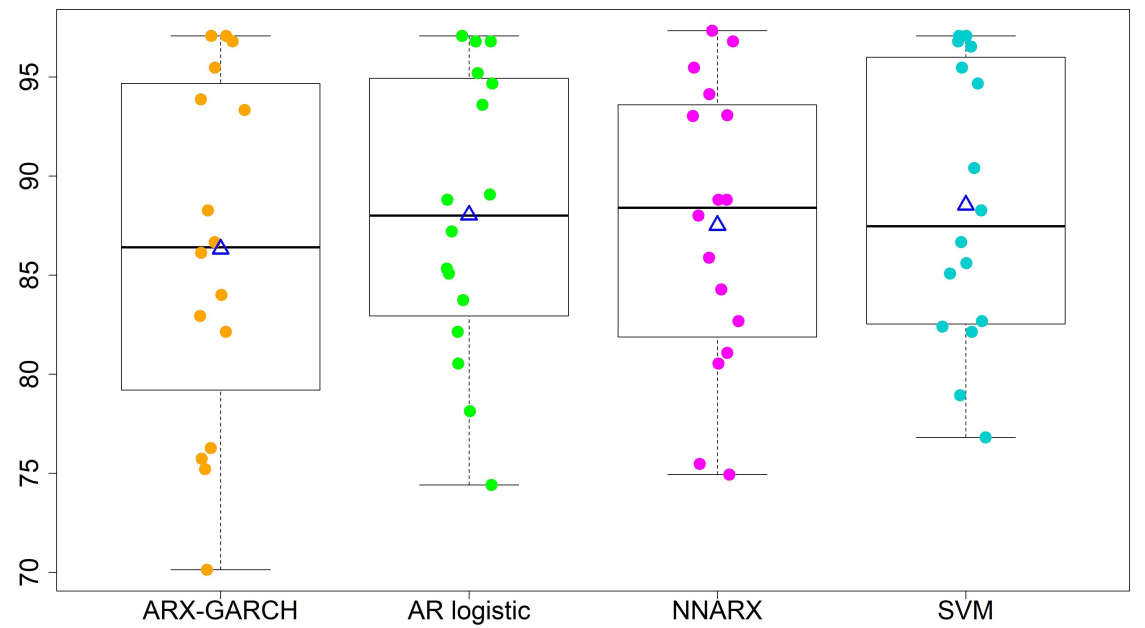

Figure 8. Forecasting performance for four-level classifications. " $\triangle$ " denotes the average of accuracy rates from all 16 sites. 


\section{Conclusions}

This study predicts one-day-ahead AQI levels for 16 cities/counties in Taiwan based on training/validation of the methodology set up herein and examines forecast accuracy by considering the ARX-GARCH, AR logistic regression, SVM, and NNARX models. We assess the accuracy of these forecasting models by a rolling window approach. These models relate to lag-1 AQI values and previous day weather covariates (PRE and TEM), while WD is a time-lag effect based on the idea of nowcasting. The results demonstrate that AR logistic regression and the SVM method are the best choices for AQI-level predictions regarding the high average and low variation accuracy rates among the four forecasting techniques. This information can greatly help the authorities to take proper action for AQI-level prediction.

There are many other established tools for ordinal time series classification, and it is worthwhile to predict ordinal time series using two other recently developed methods: the distance-based approach and the bounded-count method. We leave these new techniques for forecasting ordinal time series as a future research direction.

Author Contributions: Data curation, C.W.S.C. and L.M.C.; formal analysis, C.W.S.C.; funding acquisition, C.W.S.C.; investigation, C.W.S.C.; methodology, C.W.S.C.; supervision, C.W.S.C.; validation, C.W.S.C.; visualization, C.W.S.C.; writing—original draft, L.M.C.; writing—review and editing, C.W.S.C. Both authors have read and agreed to the published version of the manuscript.

Funding: This research was funded by the Ministry of Science and Technology, Taiwan (MOST1092118-M-035-005-MY3).

Institutional Review Board Statement: Not applicable.

Informed Consent Statement: Not applicable.

Data Availability Statement: The daily AQI values supporting the reported results can be found at https:/ / data.epa.gov.tw/dataset/aqx_p_488, (accessed on 29 June 2021). The daily weather variables are available from https:/ / e-service.cwb.gov.tw /HistoryDataQuery /, (accessed on 29 June 2021).

Acknowledgments: The authors thank the academic editor and three anonymous referees for their valuable time and careful comments, which have improved this paper.

Conflicts of Interest: The authors declare no conflict of interest.

\section{Abbreviations}

The following abbreviations are used in this manuscript:

AQI Air quality index

AR logistic regression Autoregressive multinomial logistic regression

ARX

Autoregression with exogenous variables

$\mathrm{ARCH} \quad$ Autoregressive conditional heteroskedasticity

GARCH Generalized autoregressive conditional heteroskedasticity

NNARX Neural network autoregression with exogenous variables

SVM Support vector machine

\section{References}

1. Ferlito, S.; Bosso, F.; De Vito, S.; Esposito, E.; Di Francia, G. LSTM Networks for Particulate Matter Concentration Forecasting in AISEM Annual Conference on Sensors and Microsystems; Springer: Cham, Switzeland, 2019; pp. 409-415.

2. Song, C.; Fu, X. Research on different weight combination in air quality forecasting models. J. Clean. Prod. 2020, 261, 121-169. [CrossRef]

3. Wu, Q.; Lin, H. Daily urban air quality index forecasting based on variational mode decomposition, sample entropy and LSTM neural network. Sustain. Cities Soc. 2019, 50, 101-657. [CrossRef]

4. Xiao, F.; Yang, M.; Fan, H.; Fan, G.; Al-Qaness, M.A. An improved deep learning model for predicting daily $\mathrm{PM}_{2.5}$ concentration. Sci. Rep. 2020, 10, 20988. [CrossRef] [PubMed]

5. de Medrano, R.; Buen Remiro,V.; Aznarte, J.L. SOCAIRE: Forecasting and monitoring urban air quality in Madrid. Environ. Model. Softw. 2021, 143, 105084. [CrossRef] 
6. Liu, M.; Zhu, F.; Zhu, K. Modeling normalcy-dominant ordinal time series: An application to air quality level. J. Time Ser. Anal. 2021. [CrossRef]

7. Kim, S.E. Ordinal time series model for forecasting air quality index for ozone in Southern California. Environ. Model. Assess. 2017, 22, 175-182. [CrossRef]

8. Henry, R.C.; Chang, Y.; Spiegelman, C.H. Locating nearby sources of air pollution by nonparametric regression of atmospheric concentrations on wind direction. Atmos. Environ. 2002, 36, 2237-2244. [CrossRef]

9. Carbajal-Hernàndez, J.J.; Sànchez-Fernàndez, L.P.; Carrasco-Ochoa, J.A.; Martìnez-Trinidad, J.F. Assessment and prediction of air quality using fuzzy logic and autoregressive models. Atmos. Environ. 2012, 60, 37-50. [CrossRef]

10. So, M.K.P.; Chen, C.W.S.; Liu, F.C. Best subset selection of autoregressive models with exogenous variables and generalized autoregressive conditional heteroscedasticity errors. J. R. Stat. Soc. C Appl. Stat. 2006, 55, 201-224. [CrossRef]

11. Chen, C.W.S.; Gerlach, R.; So, M.K.P. Bayesian model selection for heteroskedastic models. Bayesian Econom. 2008, 23, 567-594.

12. Engle, R.F. Autoregressive conditional heteroscedasticity with estimates of the variance of United Kingdom inflation. Econometrica 1982, 50, 987-1007. [CrossRef]

13. Bollerslev, T. Generalized autoregressive conditional heteroskedasticity. J. Econom. 1986, 31, 307-327. [CrossRef]

14. Guanche, Y.; Minguez, R.; Méendez, F.J. Autoregressive logistic regression applied to atmospheric circulation patterns. Clim. Dyn. 2014, 42, 537-552. [CrossRef]

15. Jiang, P.; Dong, Q.; Li, P. A novel hybrid strategy for PM2.5 concentration analysis and prediction. J. Environ. Manag. 2017, 196, 443-457. [CrossRef] [PubMed]

16. Liu, B.C.; Binaykia, A.; Chang, P.C.; Tiwari, M.K.; Tsao, C.C. Urban air quality forecasting based on multi-dimensional collaborative support vector machines (SVM): A case study of Beijing-Tianjin-Shijiazhuang. PLoS ONE 2017, 12, e0179763.

17. Liu, B.C.; Wang, H.; Binaykia, A.; Fu, C.C.; Xiang, B.P. Multi-level air quality classification in China using information gain and support vector machine hybrid model. Nat. Environ. Pollut. Technol. 2019, 18, 697-708.

18. Hertz, J.A.; Krogh, A.S.; Palmer, R.G. Introduction to the Theory of Neural Computation; Westview Press: Boulder, CO, USA, 1991.

19. Hyndman, R.J.; Athanasopoulos, G. Forecasting: Principles and Practice, 2rd ed.; OTexts: Melbourne, Australia, 2018.

20. Cover, T. M.; Hart, P.E. Nearest neighbor pattern classification. IEEE Trans. Inf. Theory 1967, 13, 21-27. [CrossRef]

21. Torgo, L. Data Mining with R, Learning with Case Studies; Chapman and Hall/CRC: Boca Raton, FL, USA, 2020.

22. Hansen, B.E. Autoregressive conditional density estimation. J. Int. Econ. 1994, 35, 705-730. [CrossRef]

23. Weiß, C.H. Distance-based analysis of ordinal data and ordinal time series. J. Am. Stat. Assoc. 2020 115, 1189-1200. [CrossRef]

24. Hsu, C.Y.; Chiang, H.C.; Lin, S.L.; Chen, M.J.; Lin, T.Y.; Chen, Y.C. Elemental characterization and source apportionment of PM 10 and $\mathrm{PM}_{2.5}$ in the western coastal area of central Taiwan. Sci. Total Environ. 2016, 541, 1139-1150. [CrossRef]

25. Chen, C.W.S.; Hsieh, Y.H.; Su, H.C.; Wu, J.J. Causality test of ambient fine particles and human influenza in Taiwan: Age group-specific disparity and geographic heterogeneity. Environ. Int. 2018, 111, 354-361. [CrossRef] [PubMed]

26. Tseng, C.H.; Tsuang, B.J.; Chiang, C.J. et al. The relationship between air pollution and lung cancer in nonsmokers in Taiwan. J. Thorac. Oncol. 2019, 14, 784-792. [CrossRef] [PubMed]

27. Tang, K.T.; Ku, K.C.; Chen, D.Y.; Lin, C.H.; Tsuang, B.J.; Chen, Y.H. Adult atopic dermatitis and exposure to air pollutants-A nationwide population-based study. Ann. Allergy Asthma Immunol. 2017, 118 , 351-355. [CrossRef] [PubMed]

28. Ghalanos, A.; Kley, T. rugarch: Univariate GARCH Models; R Package Version 1.4-4; 2021. Available online: https://cran.r-project. org / web/packages/rugarch/index.html (accessed on 29 June 2021).

29. Ripley, B.; Venables, B.; Bates, D.M.; Hornik, K. MASS: Support Functions and Datasets for Venables and Ripley's MASS; R Package Version 7.3-54; 2021. Available online: http:/ / www.stats.ox.ac.uk/pub/MASS4/ (accessed on 29 June 2021).

30. Meyer, D.; Dimitriadou, E.; Hornik, K.; Weingessel, A.; Leisch, F. e1071: Misc Functions of the Department of Statistics, Probability Theory Group (Formerly: E1071), TU Wien, R package version 1.7-8; 2021. Available online: https://cran.r-project.org/web/ packages/e1071/index.html (accessed on 29 June 2021). 